\title{
Methods for adaptive control of objects with variable parameters
}

\author{
Maksadkhan Yakubov ${ }^{1}$, and Gulchekhra Jamalova ${ }^{2 *}$ \\ ${ }^{1}$ Tashkent University of Information Technologies named after Muhammad al-Khorezmi, Tashkent, \\ Uzbekistan \\ ${ }^{2}$ Karshi Engineering and Economic Institute, Kashkadarya, Uzbekistan
}

\begin{abstract}
The work uses the method of standard characteristic polynomials, based on the Lyapunov theorem on adaptive control systems, the theory of flexible and robust control, methods of the theory of nonlinear systems. When modeling an internal combustion engine, methods of identification theory were additionally involved. When obtaining theoretical results, the method of Lyapunov functions, the method of standard characteristic polynomials, methods of the theory of adaptive and robust control, methods of the theory of nonlinear systems were used. When constructing a model of an internal combustion engine, methods of identification theory were additionally involved.

For the synthesis of control systems in conditions of uncertainty, one of the topical directions is adaptive systems. These are control systems that compensate for parametric, signal, functional, or structural uncertainties of the control object by automatically adjusting the controller during the system's working operation, i.e., adaptive systems make up for the lack of a priori information about the control object during operational operation. To solve the problem of managing undefined objects, for example, classical methods are used. In such methods, when state variables are immeasurable, it becomes necessary to use additional dynamic filters. Classical methods are more often used for a limited class of objects. In the case of class extension, the structure of the control algorithm becomes more complicated.
\end{abstract}

\section{Introduction}

One of the current directions of the modern theory of adaptive and robust control is the search for ways to build sufficiently simple control algorithms for objects with uncertainties. By simplifying the control algorithm, we understand both the reduction of its dynamic order and the reduction of the number of arithmetic operations in the regulator structure, the reduction of the number of adjustable parameters, and the number of measurable variables. The relevance of this direction is due to the problem of complexity of existing solutions, even for simple object models.

\footnotetext{
*Corresponding author: guli.jamalova@mail.ru
} 
To state the idea underlying the method of synthesis of adaptive regulators, let us consider a simple example of the problem of stabilizing the output of a nonstationary scalar object of the form:

$$
x=\theta x+u+\delta
$$

where $\mathrm{x}$ is the controlled variable; $\mathrm{u}$ is the control signal, ( $\mathrm{t})$ is the bounded unknown function; $\delta$-perturbation $\delta \leq \delta, \delta=$ const $>0$ ).

We propose the following stabilizing control law:

$$
u=-\omega x
$$

where $\omega$ is generated according to the integral law

$$
\omega=\gamma x^{2}
$$

where $\gamma$ is a positive parameter.

To analyze the stability of a closed-loop system, let's substitute (2) into (1) and introduce the Lyapunov function

$$
V=\frac{1}{2} x^{2}
$$

and analyze its derivative: $\theta$

$$
\begin{gathered}
\dot{V}=(\theta-\omega) x^{2}+\delta x=\frac{(\theta-\omega)}{2} x^{2}+\frac{(\theta-\omega)}{2}+x^{2}+\delta x \pm \frac{1}{2(\theta-\omega)} \delta^{2}= \\
=\frac{(\theta-\omega)}{2} x^{2}-\left(\sqrt{\frac{(\theta-\omega)}{2}}-\frac{\delta}{\sqrt{2(\theta-\omega)}}\right)^{2}+\frac{\delta^{2}}{2(\theta-\omega)} \leq \frac{(\theta-\omega)}{2} x^{2}+\frac{1}{2(\theta-\omega)} \bar{\delta}^{2}
\end{gathered}
$$

or

$$
\dot{V} \leq(\theta-\omega) V+\frac{1}{2(\theta-\omega)} \bar{\delta}^{2}
$$

The result shows that when growing according to the law (3) up to a threshold above sup $\{\theta(t)\}$ the output $\mathrm{x}$ exponentially tends $\mathrm{t} 0$,

$$
t \in(0, \infty)
$$

to some bounded neighborhood of the zero equilibrium position. Moreover, since $\mathrm{x}$ will not reach zero due to the action of perturbation, by virtue $\omega$ of (3) grows unboundedly. At the same time, the radius of the neighborhood is equal to $\bar{\delta}^{2} / 2(\theta-\omega)$, is decreasing.

The presented example illustrates several properties of a closed-loop system and problems related to the realization and proof of the stability of a closed-loop system. 
First, according to (3) and due to the presence of perturbation, the parameter grows indefinitely, which prevents the practical implementation of the control law. Consequently, modifications of (3) aimed at the limitation of growth are required.

Secondly, the equations of the closed system

$$
\left\{\begin{array}{l}
\dot{x}=(\theta-\omega) x+\delta, \\
\dot{\omega}=\gamma x^{2}
\end{array}\right.
$$

are nonlinear, which in the case of arbitrary order and arbitrary relative degree of the object requires an analysis of the completeness of the system and complicates the analysis of its stability [1].

Note also that if the object is described by a multivariate nonstationary equation

$$
\dot{x}=A(t) x+b(t) u+\delta
$$

then an extension of the structure of the control algorithm (2), (3) is required. Moreover, simple solutions will be of the greatest interest, the minimum number of algebraic operations in its implementation. Thus, the main subject of research reflected in the article is to obtain a simple, practically realizable solution of the control problem for a nonstationary parametrically indeterminate object of arbitrary order and arbitrary relative degree.

\section{Methods}

In obtaining theoretical results, we used the Lyapunov function method, the method of standard characteristic polynomials, methods of the theory of adaptive and robust control, methods of the theory of nonlinear systems. When constructing a model of an internal combustion engine, methods of identification theory were additionally used.

For the synthesis of control systems in conditions of uncertainty, adaptive systems are one of the topical directions. They are such control systems that provide compensation of parametric, signal, functional, or structural uncertainties of the control object due to automatic adjustment of the regulator in the course of operation of the system, i.e., adaptive systems compensate for the lack of a priori information about the control object in the course of operation [1].

For example, classical methods are used to solve the problem of control of uncertain objects. In such methods with unmeasurable state variables, it is necessary to use additional dynamic filters. Classical methods are more often used for a limited class of objects. In the case of class expansion, the structure of the control algorithm becomes more complicated. Let us take a closer look at some methods.

\subsection{Adaptive piecewise linear control methods}

In classical piecewise linear control (Adaptive Gain-Scheduling Control) [2, 3], the controller synthesis is based on a local description of nonlinear systems [4]. The local properties of a nonlinear system can most often be approximated to those of a linear system. Therefore, the synthesis of a local controller can be performed using the classical theory of linear systems. Such a controller guarantees that the specified requirements are met only when the local models are valid. The main results in this direction have been obtained by Andy Packard [5], Pierre Apkarian [6], Jeff Shamma [7], etc. 
Control methods for a class of linear systems with variable parameters. Linear Parameter Varying Systems (in the English-language literature, Linear Parameter Varying systems (LPV)) [4, 8] allow us to greatly simplify the problem of nonlinear modeling by replacing a nonlinear dynamic system with a linear one. Another approach is to solve systems with variable parameters using linear matrix inequalities. It is based on the Lyapunov theory. The analysis and synthesis of systems with variable parameters, using the method of linear matrix inequalities (LMI), is presented in [9].

\subsection{Methods of classical adaptive control}

Another approach for solving a control problem with uncertainties is the use of adaptive systems. They are used when there is insufficient a priori information about the object or when the object parameters change significantly during the process of functioning. The main goal of such systems is to compensate for the parametric uncertainty. Various methods are used to solve this problem. For example, the method of indirect adaptive control. This method consists of constructing a separate identifier block, which sets the parameters of the object model, and provides information support for the controller.

The direct adaptive control method consists of constructing an adaptation algorithm based on minimizing the control error. In adaptive systems with a reference model, the controller adapts so that the motion of the closed-loop system coincides with the motion of the reference model with the highest possible accuracy. Most often, the reference model is implemented as a linear system that forms the desired response to the controlling action. The adjustable regulator is constructed so that when its coefficients match the parameters of the control object, the closed-loop system behaves exactly the same as the reference model.

In the adaptation method with a "strong" feedback coefficient, the object is closed looped by linear feedback and has a "large gain." For example, [10] discusses the adaptive control method, where the main idea is that the feedback coefficient is adjusted according to the selected Lyapunov function. The gain in the controller is not monotonically nondecreasing and grows or decreases depending on the derivatives introduced.

Such scientists as Annaswamy A.M., Blanchini F., Ilchmann A. were engaged in researches in this direction.

Ilkhman worked in the direction of developing a method of large amplification factors; his method is called "funnel control" in English-language literature. The main idea of this approach is that by changing the gain, the stability of the control system is achieved. Thus, the gain increases when the absolute error reaches a bounded function and decreases when the error value is separated from this bounded function.

In [11], a regulator is presented where the parameters are limited by a region in the form of a tube for nonlinear multichannel systems with an arbitrary but known relative degree greater than one for a class of objects with a finite dimension of linear systems of nonlinear systems with delay and systems with a hysteresis. The control problem is solved using an iterative procedure for generating the feedback coefficient.

The pacification method is to ensure the passivity of the system using feedback. The problem of stabilizing the controlled system will be solved using the following two-stage procedure. At the first stage, the problem of pacification of a nonlinear system is posed, which consists of ensuring the passivity of the original system by introducing state feedback and, possibly, choosing a suitable output function [16-20]. At the second stage, under additional conditions such as observability, the problem of stabilization of the passive system obtained as a result of the first stage is solved. Thus, the pacification problem, in this case, appears as an intermediate one in the synthesis of stabilizing control for nonlinear systems. 
The "sequential compensator" method is an extended type of pacification for output control of objects under conditions of parametric uncertainty. It is based on the strong feedback method, which consists of stabilizing strictly minimum phase objects using a large proportional controller. In [12], the case is considered when compensation is performed with a relative degree of more than one by introducing an additional filter on the output variable, and stabilization is achieved by increasing the regulator coefficients.

It can be concluded that the considered methods for solving the problem of controlling objects with unknown parameters are difficult to implement and have a high dynamic order.

Thus, to build an effective control system, it is important to simultaneously improve the accuracy of the model and ensure the simplicity of the derivation of the control law. Simplification is understood as a decrease in the dynamic order in the structure of the regulator, a decrease in the number of adjustable parameters, and, if possible, a decrease in the number of measured variables.

In this regard, a tendency has arisen in the theory of adaptive systems to develop relatively simple control algorithms.

The prospect of this direction is explained by the problem of the complexity of the available solutions, even for simple models of objects.

\section{Results and Discussion}

In this paper, we propose a solution to the problem of adaptive and robust control using a simple adaptation algorithm.

Consider in the general case a nonlinear parametrically uncertain object

$$
\begin{aligned}
& \dot{x}=A(t) x+B(t) u=\xi, x(0), \\
& y=x_{1},
\end{aligned}
$$

Where $x \in R n$ is the state vector of the object; $\mathrm{u}$ is the control variable; $\mathrm{y}$ is the controlled variable; $\xi \in R n$ is the perturbation;

$$
A(t)=\left[\begin{array}{ccccc}
a_{1,1}(t) & 1 & 0 & \cdots & 0 \\
a_{1,2}(t) & a_{2,2}(t) & 1 & \cdots & 0 \\
\vdots & \vdots & \vdots & \ddots & \vdots \\
a_{1, n-1}(t) & a_{2, n-1}(t) & a_{3, n-1}(t) & \cdots & 1 \\
a_{1, n}(t) & a_{2, n}(t) & a_{n, p}(t) & \cdots & 0
\end{array}\right] ; \quad B(t)=\left[\begin{array}{c}
0 \\
\vdots \\
0 \\
b_{m}(t) \\
\vdots \\
b_{0}(t)
\end{array}\right]
$$

The parameters $a_{i}, j, i=1, n, j=1, n, b_{k}, k=0$ are unknown and may depend, among other things, including state variables $x_{i}$. The class of objects (5) is restricted to by the following assumptions:

Assumption 1: The relative degree of the object $\mathrm{p}=\mathrm{n}-\mathrm{m}$ is constant.

Assumption 2: The object is fully controllable and observable;

Assumption 3: The zero-dynamics of the object represented by the input-output equation 


$$
B(s, t)[w]=s^{m}\left[b_{m}(t) w\right]+s^{m-1}\left[b_{m-1}(t) w\right]+\ldots+s\left[b_{1}(t) w\right]+b_{m}(t) w=0
$$

where $B(s, t)$ is a right polynomial differential operator; $s=d / d t s=d / d t$ or the input-state-output equation

$$
\left[\begin{array}{c}
\dot{w}_{1} \\
\dot{w}_{2} \\
\vdots \\
\dot{w}_{m}
\end{array}\right]=\left[\begin{array}{ccccc}
-b_{m-1}(t) / b_{m}(t) & 1 & 0 & \cdots & 0 \\
-b_{m-2}(t) / b_{m}(t) & 0 & 1 & \cdots & 0 \\
\vdots & \vdots & \vdots & \ddots & \vdots \\
-b_{1}(t) / b_{m}(t) & 0 & 0 & \cdots & 1 \\
-b_{0}(t) / b_{m}(t) & 0 & 0 & \cdots & 0
\end{array}\right]\left[\begin{array}{c}
w_{1} \\
w_{2} \\
\vdots \\
w_{m}
\end{array}\right]
$$

is exponentially stable, i.e. the object is minimally phase-invariant.

In [1], it is shown that from the exponential stability of the reduced linear system follows the existence of the Lyapunov function

$$
V=w^{T} P(t) w
$$

Where $P(t)=P^{T}(t)>c_{1} I$ as $P(t)+A_{b}^{T}(t) P(t)+P(t) A_{b}(t)=-Q(t)$; $Q(t)=Q^{T}(t)>c_{2} I ; c_{1}, c_{2}$ - some positive constants;

$$
A_{b}(t)=\left[\begin{array}{ccccc}
-b_{m-1}(t) / b_{m}(t) & 1 & 0 & \cdots & 0 \\
-b_{m-2}(t) / b_{m}(t) & 0 & 1 & \cdots & 0 \\
\vdots & \vdots & \vdots & \ddots & \vdots \\
-b_{1}(t) / b_{m}(t) & 0 & 0 & \cdots & 1 \\
-b_{0}(t) / b_{m}(t) & 0 & 0 & \cdots & 0
\end{array}\right]
$$

such that V satisfies the conditions of exponential stability [1]:

$$
\begin{gathered}
k_{1}\|w\|^{2} \leq w^{T} P(t) w \leq k_{2}\|w\|^{2} ; \\
-w^{T} Q(t) w \leq-k_{3}\|w\|^{2}
\end{gathered}
$$

$K_{1}, K_{2} K_{3 \square}$ are positive constants ${ }^{1}$;

Assumption 4: $a_{i} j(t) b_{k}(t) \in L_{\infty} C^{\infty}$

Assumption 5: The minimum value of $b_{m}$, is known, denoted as $b_{m}$, min ;

Assumption 6: The state vector $x$ is directly measurable;

Assumption 7: $\|\xi\| \leq \bar{\xi}$;

Note that there is no requirement for the stability of the object. The goal is to construct a control law that ensures that in a closed system, all signals are bounded, and the following target inequality is satisfied: 


$$
\left\|y_{m}(t)-y(t)\right\| \leq \Delta, \forall t \geq T
$$

where $\Delta>0$ and $T>0$ - maximum error and control system tuning time, respectively; $y_{M} \in R^{n}$ - the desired output of the object generated by the reference model of the form

$$
\begin{aligned}
& \dot{x}_{M}=A_{M} x_{M}+B_{M} g ; \\
& y_{M}=C_{M}{ }^{x}{ }^{x}
\end{aligned}
$$

$g(t)$ is setting influence;

$$
A_{M}=\left[\begin{array}{ccccc}
0 & 1 & 0 & \cdots & 0 \\
0 & 0 & 1 & \cdots & 0 \\
\vdots & \vdots & \vdots & \ddots & \vdots \\
0 & 0 & 0 & \cdots & 1 \\
-a_{M 0} & -a_{M 1} & -a_{M 2} & \cdots & -a_{M n-1}
\end{array}\right] ; B_{M}=\left[\begin{array}{c}
0 \\
0 \\
\vdots \\
0 \\
a_{M 0}
\end{array}\right] ; C_{M}^{T}=\left[\begin{array}{c}
1 \\
0 \\
\vdots \\
0 \\
0
\end{array}\right]
$$

$a_{M i}, i=\overline{0, n-1}$ are parameters of the reference model, by means of which the desired dynamic characteristics of the closed-loop system are set. The control algorithm to be synthesized must provide the possibility of reducing the value in an arbitrary way by changing the coefficients of the regulator.

The formulated problem is broken down and solved on the basis of private solutions of control problems for narrower classes of objects. Within each particular problem, issues of setting the structure of the regulator, analysis of completeness and stability of the closedloop system, as well as ways to improve its accuracy are touched upon. A number of alternative solutions for the synthesis of adaptation algorithms that provide tuning of the regulator are considered and analyzed separately.

First of all, the partial problems of stabilization of the state vector are considered and solved, after which the transition to the solution of the desired tracking problem is made. Stabilization problems are solved sequentially for subclasses of objects of the form (5) with the following matrices $\mathrm{A}$ and $\mathrm{B}$ :

$$
\text { 1. } A=\left[\begin{array}{ccccc}
a_{1,1} & 1 & 0 & \cdots & 0 \\
a_{1,2} & a_{2, n} & 1 & \cdots & 0 \\
\vdots & \vdots & \vdots & \ddots & \vdots \\
a_{1, n-1} & a_{2, n-1} & a_{3, n-1} & \cdots & 1 \\
a_{1, n} & a_{2, n} & a_{3, n} & \cdots & a_{n, n}
\end{array}\right], B=\left[\begin{array}{c}
0 \\
0 \\
\vdots \\
0 \\
b_{0}
\end{array}\right]
$$

where $a_{i, j}, b_{0}$ are unknown constants. Within the framework of the given class of objects we consider the solution and analysis of a closed system for the case when $b_{0}-1$ and $b_{0} \geq b_{0} \min >0$; 


$$
\text { 2. } A=\left[\begin{array}{ccccc}
a_{1,1} & 1 & 0 & \cdots & 0 \\
a_{1,2} & a_{2,2} & 1 & \cdots & 0 \\
\vdots & \vdots & \vdots & \ddots & \vdots \\
a_{1, n-1} & a_{2, n-1} & a_{3, n-1} & \cdots & 1 \\
a_{1, n} & a_{2, n} & a_{3, n} & \cdots & a_{n, n}
\end{array}\right], B=\left[\begin{array}{c}
0 \\
\vdots \\
0 \\
b_{m} \\
\vdots \\
b_{0}
\end{array}\right]
$$

where $a_{i, j}, b_{j}$ are unknown constants; $b_{m} \geq b_{m \text { min }}>0$;

$$
\text { 3. } A(t)=\left[\begin{array}{ccccc}
a_{1,1}(t) & 1 & 0 & \cdots & 0 \\
a_{1,2}(t) & a_{2,2}(t) & 1 & \cdots & 0 \\
\vdots & \vdots & \vdots & \ddots & \vdots \\
a_{1, n-1}(t) & a_{2, n-1}(t) & a_{3, n-1}(t) & \cdots & 1 \\
a_{1, n}(t) & a_{2, n}(t) & a_{n, p}(t) & \cdots & 0
\end{array}\right], \quad B(t)=\left[\begin{array}{c}
0 \\
\vdots \\
0 \\
b_{m}(t) \\
\vdots \\
b_{0}(t)
\end{array}\right]
$$

where $a_{i, j}, b_{j}$ is unknown time functions; $b_{m} \geq b_{m \text { min }}>0$;

It is assumed that assumptions 2-7 are assumed in the partial problems.

\section{Conclusions}

The paper develops a method of adaptive control of nonstationary parametrically uncertain linear objects with a triangular state matrix and an arbitrary relative degree. The control law has a simple structure, which consists in a relatively low dynamic order of the controller equal to one.

This paper proposes a solution to the problem of adaptive and robust control using a simple adaptation algorithm.

\section{References}

1. Nikiforov, V.O. Upravleniye v usloviyakh neopredelennosti: chuvstvitel'nost', adaptatsiya, robastnost'. SPb GITMO (TU),p. 232. (2002)

2. Rugh, W.J. Analytical framework for gain scheduling. IEEE Contr.Sys. Mag. 11(1). pp.79- 84 .

3. Wu, F. Gain-scheduling control of LFT systems using parameter dependent Lyapunov functions. Automatica. 42 (1), pp. 39-50, (2006).

4. Bruzelius, F. Linear Parameter-Varying Systems an approach to gain scheduling. Chalmers University of Technology, (2004).

5. Packard, A. K. Gain scheduling via linear fractional transformations. Systems Control Letters. 22. pp. 79 - 92. (1994)

6. Apkarian, P. Advanced gain-scheduling techniques for uncertain systems. IEEE Control Systems Society. 6(1). pp. 21-32. (1997)

7. Rugh, W.J. Research on gain scheduling. Automatica. 36 (10), pp. $1401-1425$. (2000)

8. Ilchmann, A. A behavioral approach to time-varying linear systems. SIAM Journal of Control Optimization. 44 (5). pp. 1725 - 1747, (2005) 
9. Apkarian, P. Self-scheduled $H \infty$ control of linear parameter-varying systems. Automatica. 31. pp. 1251 - 1261. (1995)

10. Blanchini, F. High-gain adaptive control: A derivative-based approach. IEEE Decisionopmation and Control. pp. 3233 - 3238, (2008)

11. Ilchmann, A. Tracking control with prescribed transient behavior for systems of known relative degree. Systems and control letters. 55. pp. 396 - 406. (2006)

12. Bobtsov, A.A. Upravleniye po vykhodu nelineynymi sistemami s zapazdyvaniyem $\mathrm{v}$ usloviyakh neuchtennoy dinamiki. Izvestiya RAN. Teoriya i sistemy upravleniya. 3. pp. 79-87. (2011)

13. Yakubov M.S, Jamalova G.B. Build an adaptive control system with flexible functions. JournalNX-A Multidisciplinary Peer Reviewed Journal. 6(10), pp. 393396. (2020)

14. Zhamalova G.B. Informatsionnoye modelirovaniye s primeneniyem iskusstvennykh neyronnykh setey. Academic research in educational sciences, 1(3), pp. 729-742. (2020)

15. Bazarov D. and Vokhidov O. Extinguishing Excess Flow Energy in Spillway Structures. In book: Proceedings of EECE 2020, LNCE 150, pp. 535-545, (2021) DOI: 10.1007/978-3-030-72404-7_52

16. Mallayev A.R., Khusanov S.N. Algoritmy sinteza adaptivnogo suboptimal'nogo regulyatora dlya stokhasticheskogo obekta upravleniya. Materialy mezhdunarodnoy nauchnoy konferentsii «Innovatsionnyy resheniya inzhenerno-tekhnologicheskikh problem sovremennogo proizvodstva». Bukhara. pp. 237-240. (2019)

17. Shokirov B., Norkulov B., Nishanbaev Kh., Khurazbaev M., Nazarov B. Computer simulation of channel processes. E3S Web of Conferences, 97, 05012, (2019)

18. Bazarov D., Markova I., Sultanov S. and Kattakulov F. Dynamics of the hydraulic and alluvial regime of the lower reaches of the Amudarya after the commissioning of the Takhiatash and Tuyamuyun hydrosystems. IOP Conf. Ser. Mater. Sci. Eng. 1030, 012110 (2021).

19. Uralov B., Rakhmatov N., Khidirov S., Uljaev F., Raimova I. Hydraulic modes of damless water intake. IOP Conf. Ser. Mater. Sci. Eng. 1030(1), 012123 (2021)

20. Matyakubov B., Begmatov I., Raimova I. and Teplova G. Factors for the efficient use of water distribution facilities. IOP Conf. Ser. Mater. Sci. Eng. 883, 012025 (2020). 